\title{
Bleomycin-induced interstitial pneumonia in dogs
}

\author{
ROBERT W. FLEISCHMAN, JOHN R. BAKER, \\ GEORGE R. THOMPSON, ULRICH H. SCHAEPPI, \\ VLADIMIR R. ILLIEVSKI, DAVID A. COONEY, \\ and R UTH D. DA VIS
}

Mason Research Institute, Harvard Street, Worcester, Massachusetts 01608, U.S.A.

\begin{abstract}
The intravenous administration of Bleomycin to 10 dogs at different dosages resulted in varying degrees of interstitial pneumonia in all cases and a lower incidence of nephrosis, foot pad excoriation and ulceration, onychoptosis, and alopecia. Pulmonary changes did not occur as a simple dose-related phenomenon. The lesions required at least 38 days to become apparent and appeared to increase in severity with time. Even at the lowest dose used $(0.625 \mathrm{mg} / \mathrm{kg}$ body weight) very severe changes were seen 128 days after cessation of therapy. Morphological features of interstitial pneumonia were subpleural localization, focal mesothelial hyperplasia, marked hyperplasia and metaplasia of type II pneumocytes, fetalization of alveoli, and a pleomorphic inflammatory infiltrate. In cross-sections of lung lobes selected for histology approximately 1 to $22 \%$ of the parenchyma contained lesions. Involved areas showed marked elastosis, excess of reticular fibres, fibrosis, and increased acid mucopolysaccharides. The administration of Bleomycin produced pulmonary changes similar in many respects to those reported in busulphan-treated patients and desquamative interstitial pneumonia. The finding of interstitial pneumonia and pulmonary fibrosis in dogs treated with low doses over prolonged periods points the need to monitor pulmonary function in humans treated with Bleomycin.
\end{abstract}

Bleomycin, ${ }^{1}$ a polypeptide antibiotic from Streptomyces verticillus (Umezawa, 1965), showed potent activity against squamous-cell carcinoma (Tsubaki, Suzuki, and Ogawa, 1968 ; Ichikawa, 1969 ; Ichikawa, Nakano, and Hirokawa, 1969 ; Oka et al., 1969; Suzuki, Murai, Watanabe, and Nunokawa, 1970). The compound, in contrast with most antitumour agents, did not produce toxic effects on bone marrow, lymphoid tissues or the liver. In man, toxic side-effects included pulmonary fibrosis and interstitial pneumonia (Ichikawa et al., 1969 ; Tsubaki et al., 1968). A low incidence of pulmonary toxicity was reported, but several patients died from pulmonary fibrosis. Interstitial pneumonia was accompanied by slight cough, fever, leucocytosis, and radiographic infiltration, particularly in the lower lobes of the lung. Other side effects in man included anorexia, fever, nausea, vomiting, headache, stomatitis, hyperpigmentation, sclerosis, and swelling of the fingers, palms, and soles.

Pulmonary fibrosis in dogs was reported by Ishizuka, Takayama, Takeuchi, and Umezawa (1967) and by Umezawa, Ishizuka, Maeda, and 1Nippon Kayaku Co. Ltd., New Kaijo Building, 2,1-chome
Marunochi Chiyoda-ku, Tokyo, Japan (see Jap. med. Gaz., 7, 9-14 (1970)
Takeuchi (1967); however, few morphological details are available in the literature (Nippon Kayaku Co. Ltd., 1968).

\section{METHODS}

THE Japanese preparation of Bleomycin (NSC-125 066) was obtained from the Laboratory of Toxicology at the National Cancer Institute, Bethesda, Maryland (1964, 1969).

Initial studies were performed on 12 conditioned beagles 10 to 15 months old and weighing 7 to $9 \mathrm{~kg}$ obtained from commercial sources 1 . The dogs were individually housed in suspended steel cages, fed a commercial dog chow (Kibble with beef) ${ }^{2}$, and provided with water ad libitum. Sex-paired dogs were injected intravenously with Bleomycin in doses of 5.0 , $2.5,1.25$, and $0.625 \mathrm{mg} / \mathrm{kg}$ body weight every fourth day for 11 treatments or with $0.625 \mathrm{mg} / \mathrm{kg}$ body weight for 31 treatments. Bleomycin was dissolved in saline immediately before every injection. Two dogs served as controls and were treated with equivalent volumes of saline. Doses were adjusted weekly according to the weight of the animals. During the course of treatmènt physical examinations were made daily, body weight and urine samplings were per-

1G:Iman Marshall, North Rose, N.Y. Hazelton-Saunders, Inc., P.O. Box 8, Midlothian, Va.

Mother Hubbard 
T A B L E I

BLEOMYCIN EXPERIMENTAL PROTOCOL AND MAXIMUM DRUG-INDUCED TOXICITY

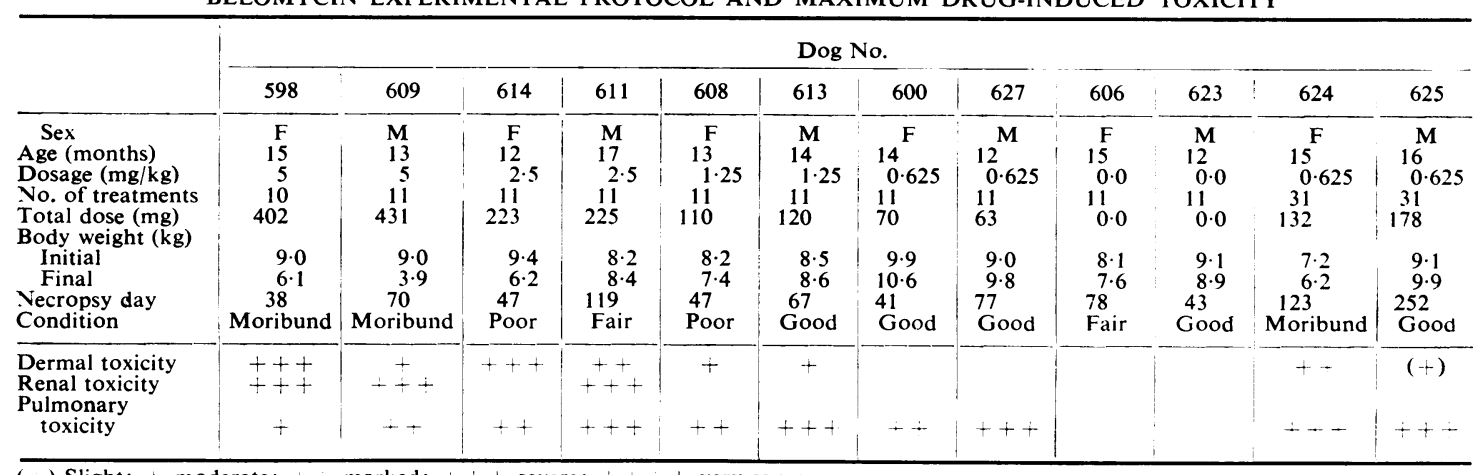

$(-)$ Slight; + moderate; ++ marked; +++ severe; ++++ very severe.

formed weekly, and lateral and dorsoventral radiographs were taken twice weekly. In addition, before and during treatment, complete haematological and blood chemical analyses were performed and are reported elsewhere (Thompson et al., 1970).

All animals were ultimately submitted to necropsy and tissues were collected for gross and histopathological evaluation. The lungs were distended with neutral buffered formaldehyde until they reached approximately normal size, the trachea was tied with string and, when fixation was complete, representative blocks were taken from each lobe. Tissues were fixed in neutral buffered formaldehyde and embedded in paraffin. Sections were cut at $6 \mu$ and stained with haematoxylin and eosin. Selected tissues were stained or reacted with picro-Mallory trichrome, celestin bluevan Gieson, aldehyde fuchsin, periodic acid-Schiff (PAS), Gram, alcian blue, colloidal iron, and silver diammino-hydroxide.

\section{RESULTS}

Three of 10 Bleomycin-treated dogs became moribund. Two dogs treated with $5 \mathrm{mg} / \mathrm{kg}$ body weight

T A B L E I I

CLINICAL SIGNS IN 10 BLEOMYCIN-TREATED DOGS

\begin{tabular}{|c|c|c|c|c|}
\hline Clinical Sign & & $\begin{array}{c}\% \\
\text { Showing } \\
\text { Sign }\end{array}$ & $\begin{array}{l}\text { Earliest } \\
\text { Day of } \\
\text { Onset }\end{array}$ & $\begin{array}{c}\text { Showing } \\
\text { Sporadic or } \\
\text { Reversible Signs }\end{array}$ \\
\hline $\begin{array}{l}\text { Weight loss } \\
\text { Foot-pad ulceration } \\
\text { Anorexia } \quad . . \\
\text { Vomiting } \\
\text { Dehydration } \quad \ldots \\
\text { Diarrhoea } \\
\text { Purulent conjunctivitis } \\
\text { Green urine .. } \\
\text { Onychoptosis } . . \\
\text { Dermatitis tail tip } \\
\text { Ulcerative stomatitis } \\
\text { Inactivity } \\
\text { Abdominal tenderness } \\
\text { Purulent rhinitis } \\
\text { Alopecia } \quad . .\end{array}$ & 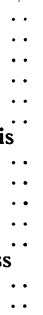 & $\begin{array}{l}80 \\
70 \\
50 \\
50 \\
40 \\
60 \\
30 \\
20 \\
20 \\
20 \\
20 \\
10 \\
10 \\
10 \\
10\end{array}$ & $\begin{array}{r}9 \\
19 \\
1 \\
6 \\
19 \\
1 \\
26 \\
34 \\
34 \\
11 \\
37 \\
19 \\
14 \\
26 \\
11\end{array}$ & $\begin{array}{r}20 \\
30 \\
10 \\
50 \\
10 \\
60 \\
10 \\
20 \\
10 \\
0 \\
0 \\
0 \\
0 \\
0 \\
0\end{array}$ \\
\hline
\end{tabular}

were moribund on days 38 and 70 . Primary toxicity in these animals included marked inanition, nephrosis, uraemia, and serum electrolyte derangements (Table I). One of two dogs treated with $0625 \mathrm{mg} / \mathrm{kg}$ body weight became moribund on day 137 and, at necropsy, exhibited an acute pneumonia superimposed on an interstitial pneumonia. All other dogs survived in good general condition. However, most of the 10 dogs exhibited dose-related pyrexia, epithelial toxicity, onychopiosis, foot-pad ulceration, dermatitis, and alopecia (Fig. 1a; Table II).

Pulmonary lesions were most severe in animals with the longest post-treatment observation periods. Macroscopic pulmonary lesions were obvious in five dogs sacrificed 70 or more days after the onset of treatment. The lesions affected 30 to $80 \%$ of the lung surface and were especially evident along the margins. They appeared as irregular, focal or confluent, slightly elevated, and firm areas of gray-tan mottling in all lobes (Fig. $1 \mathrm{~b}$ and $\mathrm{c})$. Gross pulmonary lesions were not apparent in dogs sacrificed earlier than 70 days after the onset of treatment. However, all dogs showed microscopic evidence of a focal and confluent interstitial pneumonia with a subpleural localization. Additional histopathological features were interstitial fibrosis, marked hyperplasia and metaplasia of type II pneumocytes, pleomorphic inflammatory infiltrate (Fig. 2), and focal hyperplasia of pleural mesothelium (Fig. 3). Deep parenchymal areas were entirely free of lesions. The proliferative and metaplastic changes affecting the alveolar lining cells sometimes produced a continuous cuboidal epithelium giving alveoli a similar appearance to that of the fetus. Preliminary investigations in monkeys revealed similar gross and microscopic lesions. 

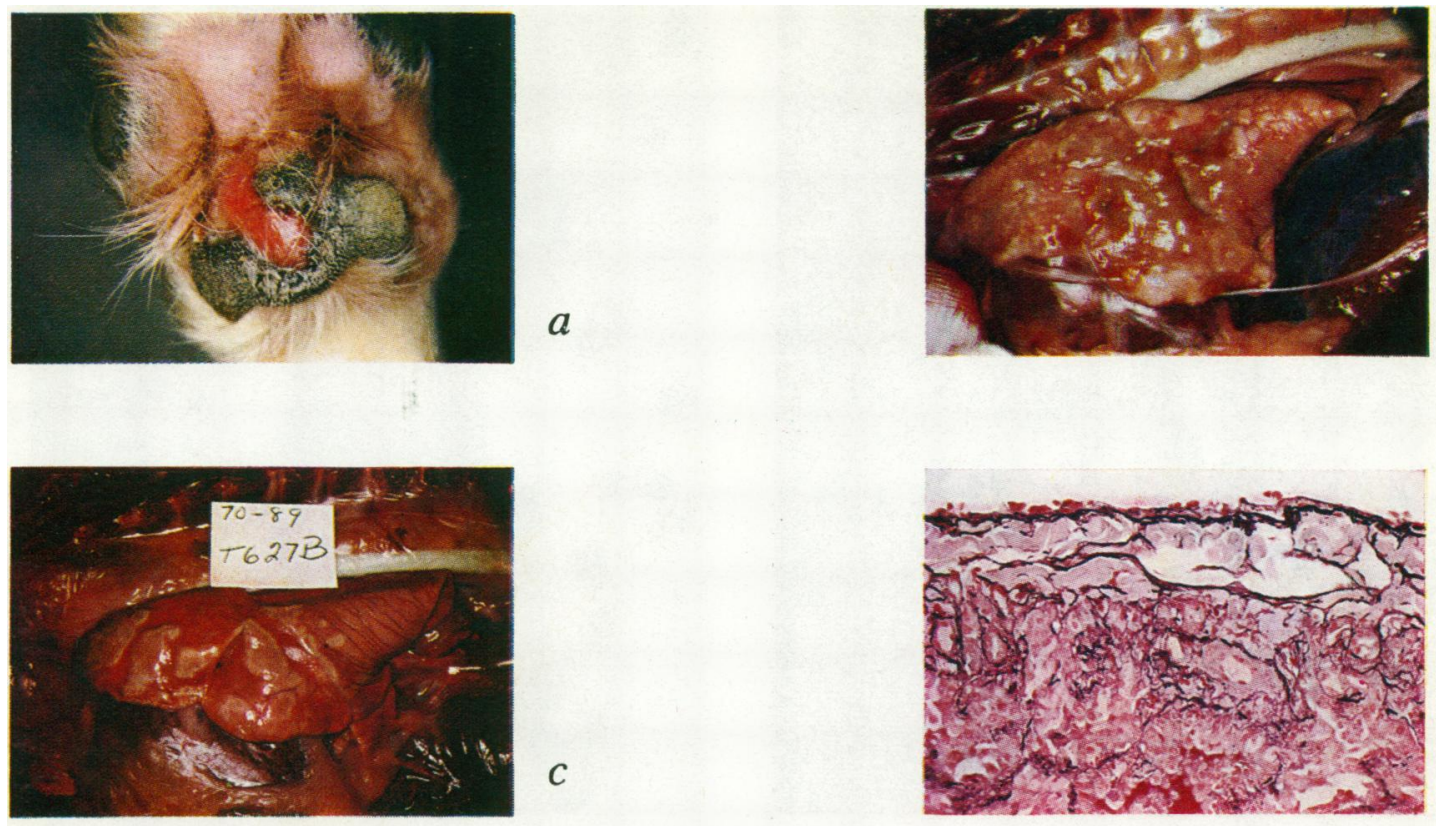

c
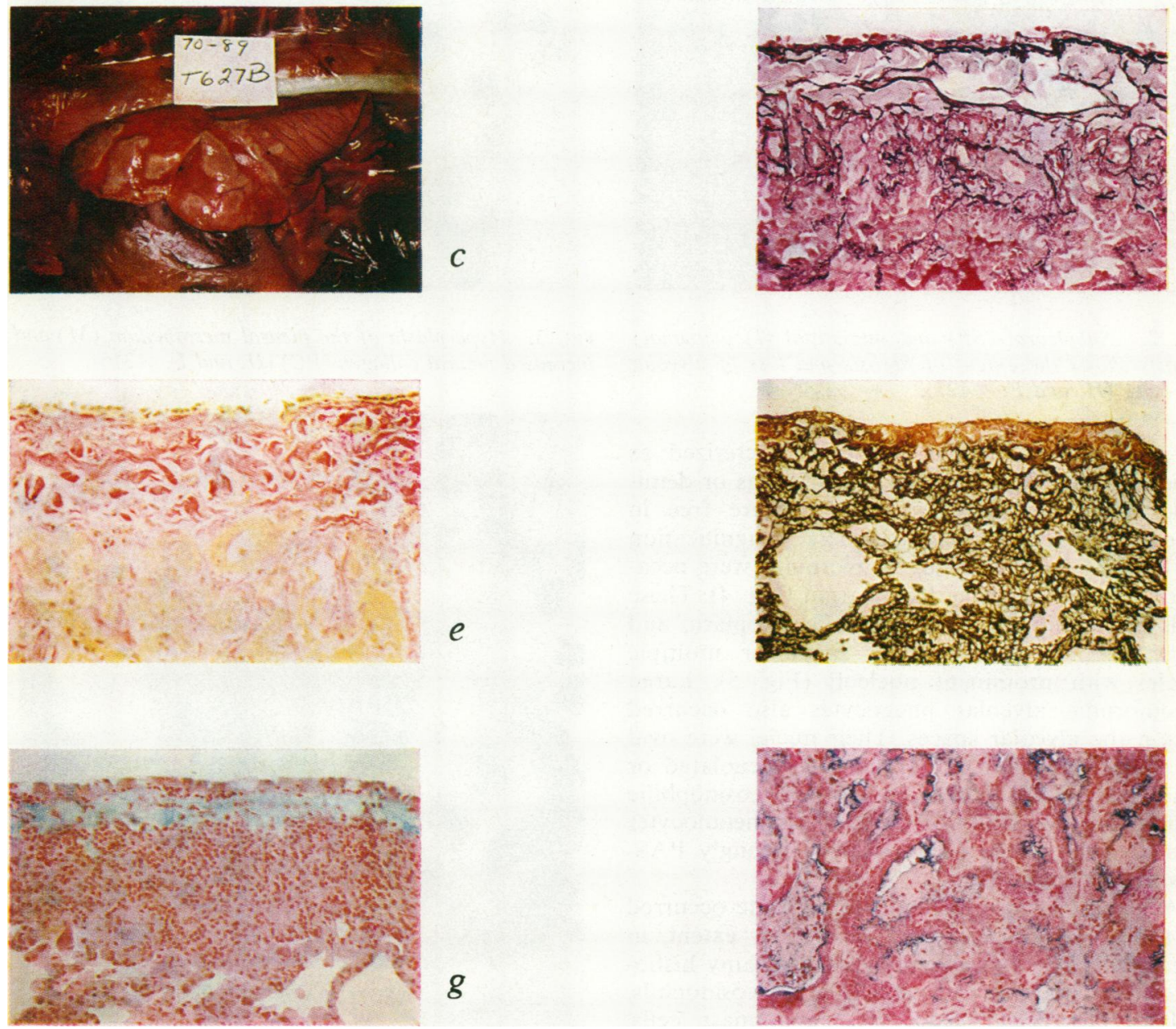

FIG. 1. (a) Dog 598. Gross appearance of ulcerated metacarpal foot pad. (b) Dog 624. Gross in situ appearance of left lung showing diffuse gray-tan mottling. (c) Dog 627. Gross in situ appearance of left lung showing discrete, slightly elevated areas of gray-tan mottling. (d) Increased pleural and interstitial elastica stained blue-black (aldehyde fuchsinneutral red $\times 160)$. (e) Increased pleural and interstitial collagen stained red (celestin blue-van Gieson $\times 160)$. $(f)$ Increased reticulin; reticulin black, collagen gold-yellow (modified Gordon and Sweet silver impregnation $\times 160)$. $(g)$ Increased pleural and alveolar acid mucopolysaccharides; polysaccharides pale blue (alcian blue-neutral red $\times 160)$. $(h)$ Increased alveolar acid mucopolysaccharides; polysaccharides deep blue (colloidal iron-neutral red $\times 160$ ). 


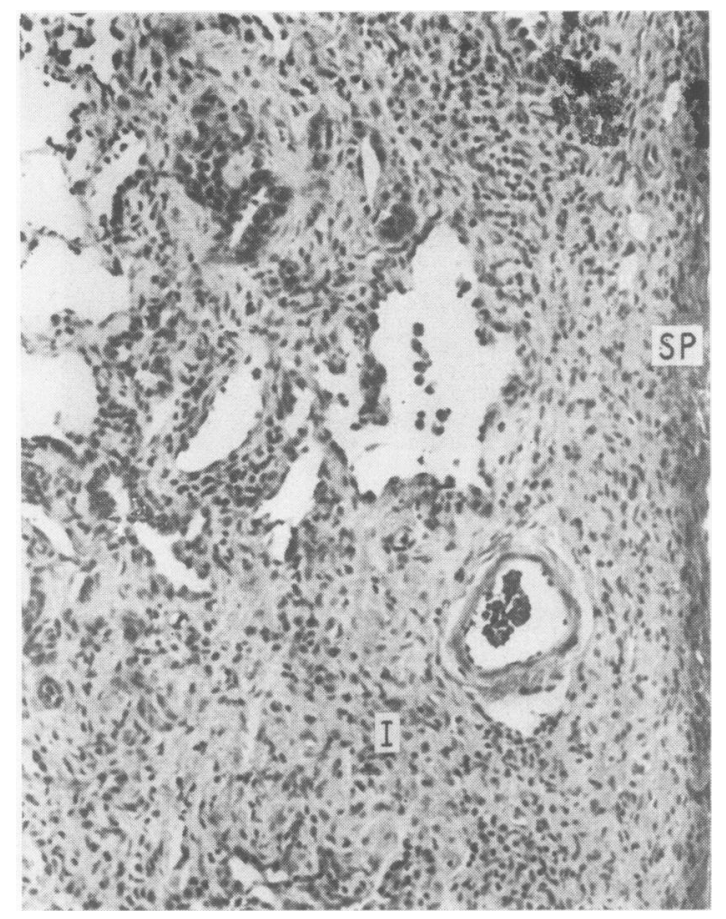

FIG. 2. Subpleural (SP) and interstitial (I) pulmonary fibrosis shows the extent of fibrosis and loss of alveolar structure $(H$. and $E . \times 125)$.

In affected areas, large cells characterized as type II pneumocytes, formed protrusions or demilunes from the alveolar walls or were free in alveolar spaces. Under highest magnification $(\times 1,000)$ regularly arranged microvilli were occasionally seen along the free margin (Fig. 4). These cells also exhibited an eosinophilic cytoplasm and large vesicular pleomorphic single or multiple nuclei with prominent nucleoli (Fig. 5). Large pleomorphic alveolar phagocytes also occurred within the alveolar spaces. Their nuclei were oval or round and their cytoplasm was vacuolated or granular, occasionally containing eosinophilic debris (Fig. 6). Many of the type II pneumocytes and alveolar phagocytes contained strongly PASpositive granules.

A pleomorphic inflammatory infiltrate occurred in the alveolar spaces and, to a lesser extent, in the interstitum. It was composed of foamy histiocytes, lymphocytes, plasma cells, a few eosinophils, polymorphonuclear leucocytes, and mast cells. Some alveoli were filled with oedema fluid while others contained organized polypoid projections covered by metaplastic pneumocytes. The core of these projections was hyalinized or fibrosed (Fig. 7). In some terminal bronchioles and alveoli there was a pronounced hyperplasia and metaplasia of the lining cells with a loss of nuclear orientation (Fig. 8).

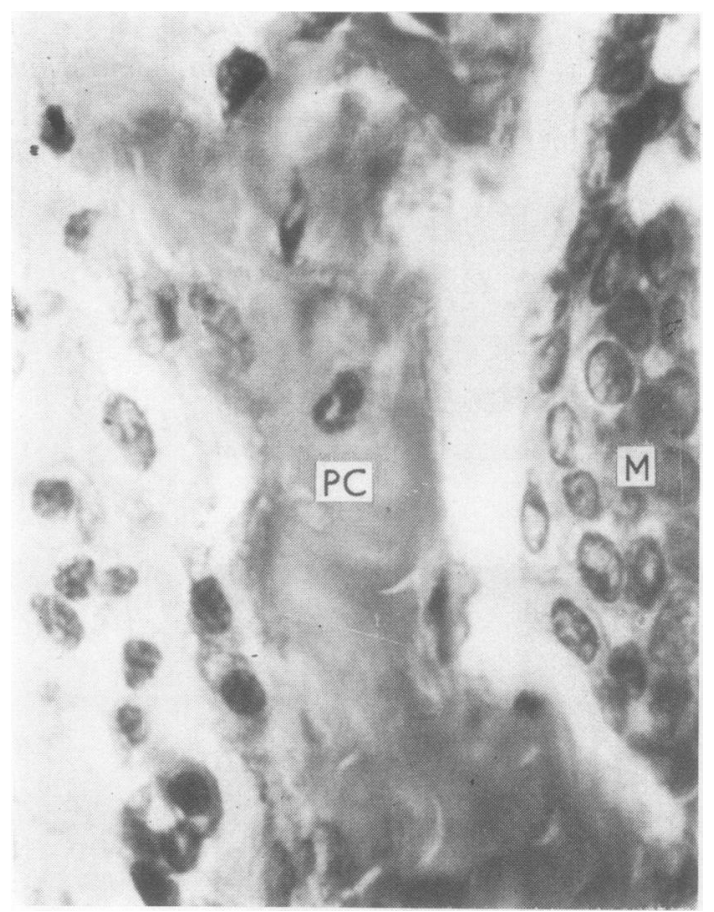

FIG. 3. Hyperplasia of the pleural mesothelium $(M)$ and increased pleural collagen $(P C)(H$. and $E$. $\times 315)$.

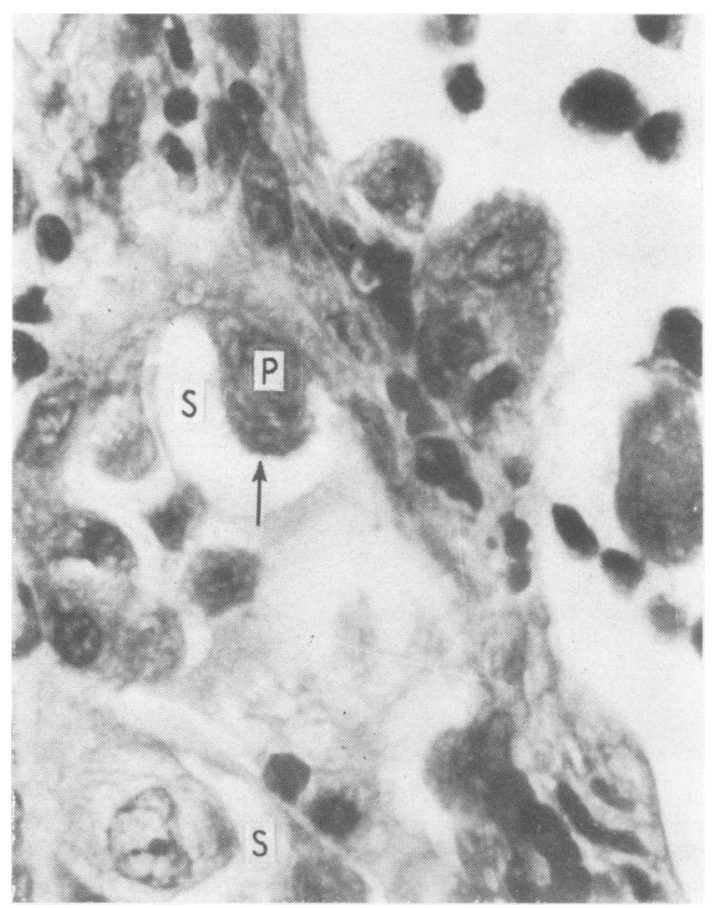

FIG. 4. Hyperplastic type II pneumocytes $(P)$, one of which shows regularly arranged microvilli (arrow); and restricted alveolar spaces $(S)(H$. and $E$. $\times 785)$. 


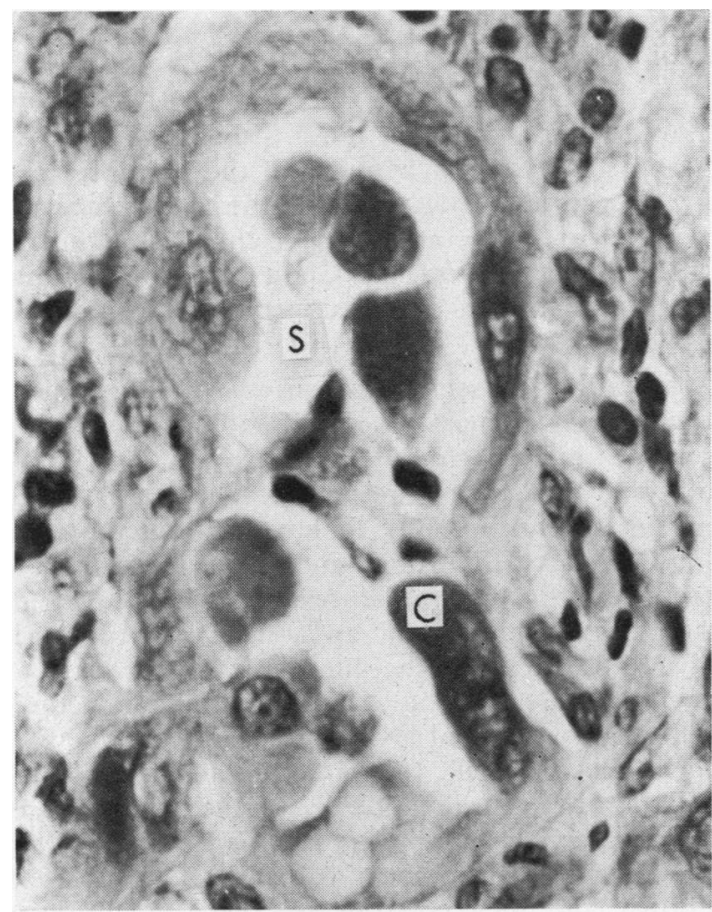

FIG. 5. Hyperplastic and metaplastic cells (C) lining a fibrosed alveolus with a reduced alveolar space (S) (H. and $E . \times 315$ ).

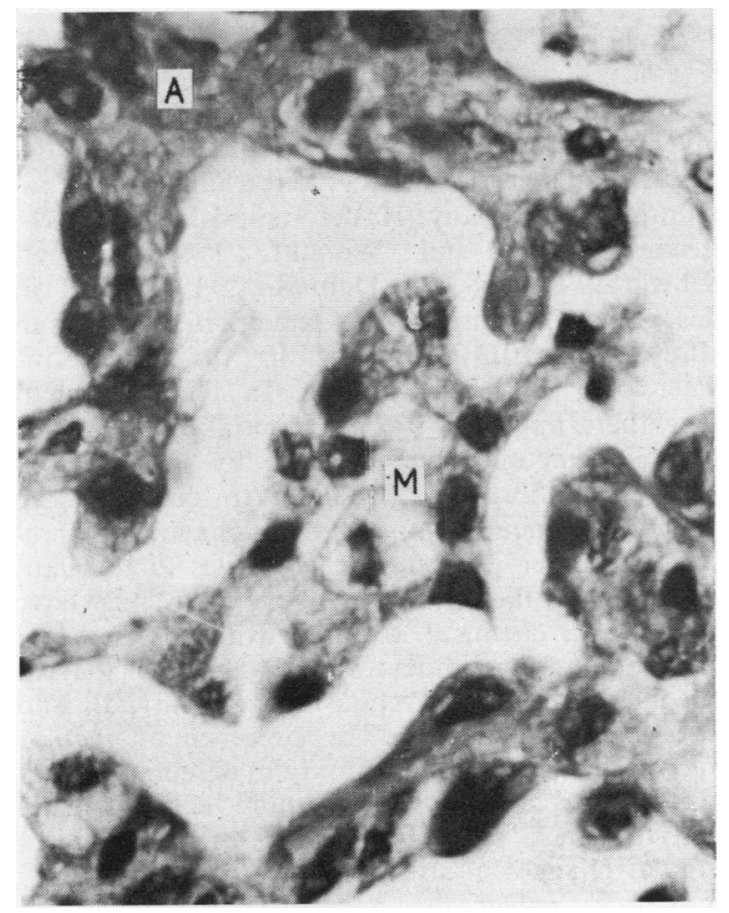

FIG. 6. Syncytial mass $(M)$ of alveolar macrophages in a fibrosed alveolus $(A)(H$. and $E . \times 315)$.

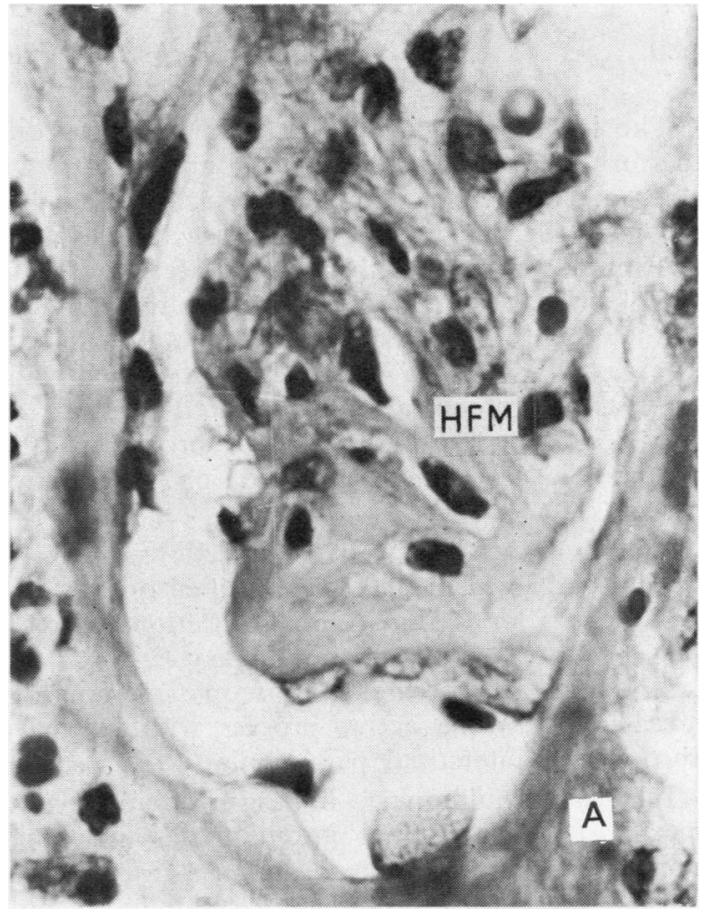

FIG. 7. A hyalinized and fibrosed mass (HFM) projecting. from the wall of a fibrosed alveolus $(A)(H$. and $E . \times 125)$

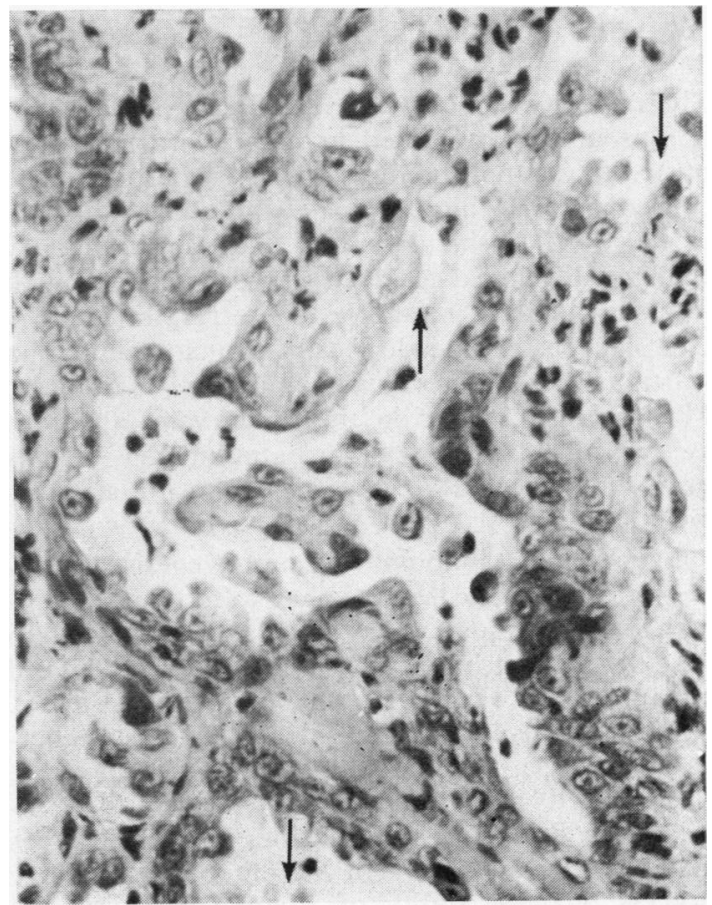

FIG. 8. Terminal bronchiole $(\uparrow)$ and adjacent alveoli $(\downarrow)$ lined by hyperplastic and metaplastic cells $(H$. and $E . \times 125)$. 
The loss of normal alveolar structure was due to an increase in subpleural and interstitial elastica, collagen, acid mucopolysaccharides, and reticulin (Fig. 1d to g). Mucopolysaccharides were distributed throughout the fibrosed areas and were prominent on the surface of proliferative alveolar epithelium (Fig. 1h). Alveoli immediately adjacent to areas of interstitial fibrosis had thickened walls whereas peripheral alveoli appeared normal. In two dogs, focal areas of acute bronchitis and pneumonia were superimposed on the above process.

The dog that survived 31 injections of 0.625 $\mathrm{mg} / \mathrm{kg}$ body weight and was sacrified on day 258 (137 days after termination of injections) exhibited the usual gray-tan pulmonary lesions and extensive white scarring. Microscopic examination revealed a chronic active process with extensive fibrosis and interstitial penumonia.

Incidental pulmonary findings included nonspecific focal interstitial granulomata and lungworms, presumably Filaroides milksi, in two dogs. In five dogs with prominent drug-induced pulmonary changes affecting all seven lobes, neither pulmonary nematodes nor interstitial granulomata were observed.

\section{DISCUSSION}

These results demonstrate that Bleomycin-induced lesions in dogs occurred in organs previously reported to contain the highest concentrations of the compound, i.e., lung, kidney, and skin (Ichikawa et al., 1967; Ishizuka et al., 1967). Pulmonary changes developed gradually and insidiously with few clinical signs referable to the respiratory system, except for one dog with bronchopneumonia which became moribund and was sacrificed. Microscopic changes in the lungs were apparent as early as $\mathbf{3 8}$ days while gross pulmonary lesions were first detected 70 days after the initial treatment.

From the standpoint of pathogenesis, it appeared that the earliest changes were focal inflammation and exudation of alveolar phagocytes. In time, thickening of the pleura and hyperplasia and metaplasia of type II pneumocytes occurred. Eventually alveoli were replaced by a considerable increase in interstitial collagen, reticulin, elastin, and acid mucopolysaccharides. The increased acid mucopolysaccharides in affected areas may have resulted from (1) the inflammatory process, (2) the ability of Bleomycin to chelate with copper or ferric ions (Umezawa, Maeda, Takeuchi, and Okami, 1966; Ichikawa et al.,
1967 ; Umezawa et al., 1967), (3) cross-linkage of Bleomycin with polypeptides of the glycoproteins, (4) increased production of the polysaccharide fraction of surfactant through changes caused in type II pneumocytes and Clara cells, or (5) functional impairment of mucus transport because of fibrosis.

Irritation caused by a variety of aetiological agents such as dusts, chemicals, tobacco particles, viruses, and bacteria may provoke an initial alveolar phagocytic response. Therefore, Bleo- N mycin appears initially to have an effect on the $\dot{\sigma}$ lung similar to that of many known irritants. The tendency for this initial response to localize in $O$

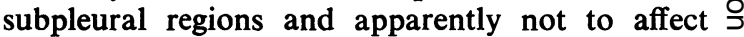
alveoli throughout the lung may be related to peculiarities in the pulmonary architecture. Although peripheral alveoli are the first to aerate, they are less well ventilated because of partial isolation by pleural connective tissue invaginations which preclude the phenomenon of collateral air drift. In human chronic bronchitis, the foci of fibrosis and lung collapse are twice as frequent subpleurally as in the deepest parenchyma, suggesting that ventilation is less effective where the lung is restricted by pleura (Reid, 1967). In addition, the distribution of skin and pulmonary lesions suggests that pressure and/or friction may also be involved in the development of these changes.

Morphologically, the changes seen in the Bleomycin lungs are similar in some respects to those described as a side effect of busulphan chemotherapy (Littler, Kay, Hasleton, and Heath, 1969; Leake, Smith, and Woodiff, 1963; Oliner, Schwartz, Rubio, and Dameshek, 1961) and in desquamative interstitial pneumonia in man (Brewer, Heath, and Asquith, 1969; Liebow, Steer, and Billingsley, 1965). In the busulphan lung there appeared to be an initial exudation of granular pneumocytes followed by the development of a fibrosing alveolitis. In desquamative interstitial pneumonia, alveolar phagocytes predominate over granular pneumocytes in the exudative phase. Bleomycin, like busulphan, may have produced a chemical alveolitis with proliferation of granular pneumocytes and alveolar macrophages followed by fibrosis of alveolar walls and intra-alveolar contents. The presence of regular microvilli along the free margin of some hyperplastic-metaplastic alveolar lining cells suggests that these are type II pneumocytes. The bizarre cellular changes in alveolar and bronchiolar lining cells may reflect the action of Bleomycin on deoxyribonucleic acid (DNA). Bleomycin appar. 
ently acts by splitting single-stranded DNA, interfering with protein synthesis, and inhibiting DNApolymerase (Nippon Kayaku Co., Ltd., 1968). Littler et al. (1969) noted that changes in type II granular pneumocytes may occur in conditions in which there is chemical or physical irritation of alveolar walls, with the production of cellular and, later, fibrosing stages of alveolitis. According to Spencer (1968), damage to the alveolar epithelium is one of several ways in which chronic interstitial fibrosis originates. The observations in alveolar lining cells in our study are compatible with this concept.

Hirth and Hottendorf (1969) have raised the problem of differentiating drug-induced pulmonary changes from those produced by Filaroides milksi in experimental dogs. Although 2 of 10 dogs showed evidence of Filaroides milksi, the complete absence of any incidental lesions in five dogs with well-developed pulmonary changes plus the consistent occurrence of similar pulmonary lesions in Bleomycin-treated monkeys (in progress) leaves no doubt that the changes are drug-induced. Furthermore, similar changes have been observed in the lung of a human patient treated with Bleomycin for a squamous-cell carcinoma of the nasopharynx (Luce, 1970).

Lesions produced in the dog lung in this study must, for the most part, be considered irreversible. Where reticulin and collagen have replaced the alveolar septum such portions of the lungs will not readily regain normal respiratory function. Should drug administration be halted at this stage, the remaining portions of the lung which show no morphological changes should retain normal function provided the processes are not progressive. Although pneumonia and pulmonary toxicity following Bleomycin therapy have been reported as reversible in man, the production of such lesions remains the most serious side effect of the drug. The finding of interstitial pneumonia and pulmonary fibrosis in dogs treated with low doses over prolonged periods indicates the need to monitor pulmonary function in humans treated with Bleomycin. The only obvious parameters for assessing the clinical stage of Bleomycin toxicity in the present study were the skin changes. One must accept that skin and pulmonary changes occur concomitantly, as evidenced by the finding of welldeveloped pulmonary lesions as early as one to eight days after the appearance of skin lesions. Further investigation will be necessary to establish a non-toxic dose in dogs and whether reversibility of early pulmonary changes is possible with adequate therapeutic measures, using the skin as the index of pulmonary involvement.
This study was supported by Contract NIH-70-2055 with Chemotherapy, National Cancer Institute, National Institutes of Health.

\section{REFERENCES}

Brewer, D. B., Heath, D., and Asquith, P. (1969). Electron microscopy of desquamative interstitial pneumonia. J. Path., 97, 317.

Hirth, R. S., and Hottendorf, G. H. (1969). A spontaneous pulmonary disease in purebred beagle dogs used in toxicity studies. Abstract. Society of Toxicology 8th Annual Meeting. Williamsburg, Virginia 66, 53.

Ichikawa, T. (1969). Bleomycin, a new antitumor antibiotic. Translation of J. Japan med. Ass., 61, 487.

— Matsuda, A., Miyamoto, K., Tsubosaki, M., Karohara, T., Sakamoto, K., and Umezawa, H. (1967). Biological studies on bleomycin. J, Antibiot. (Tokyo), 20, 149.

- Nakano, L., and Hirokawa, I. (1969). Bleomycin treated of tumors of the penis and scrotum. J. Urol. (Baltimore), 102, 699.

Ishizuka, M., Takayama, H., Takeuchi, T., and Umezawa, H. (1967). Activity and toxicity of Bleomycin. J. Antibiot. (Tokyo), 20, 15.

Leake, E., Smith, W. G., and Woodiff, H. J. (1963). Diffuse interstitial pulmonary fibrosis after Busulphan therapy. Lancet, 2, 432.

Liebow, A. A., Steer, A., and Billingsley, J. G. (1965). Desquamative interstitial pneumonia. Amer. J. Med., 39, 369.

Littler, W. A., Kay, J. M., Hasleton, P. S., and Heath, D. (1969). Busulphan lung. Thorax, 24, 639.

Luce, James K. (1970). Personal communication. M. D. Anderson Hospital and Tumor Institute, University of Texas, Houston, Texas.

National Cancer Institute (1964). Cancer Chemotherapy. Abstr., 37, 1.

- (1969). General protocol for preclinical toxicology (Laboratory of Toxicology, N.C.I., May 27).

Nippon Kayaku Co. Ltd. (1968). Synopsis on Bleomycin. New Kaijo Bldg., 2, 1-Chome Marunouchi Chiyoda-ku, Tokyo, Japan.

Oka, S., Sato, K., Nakai, Y., Awata, K., Hashimoto, K., and Oshibe, M. (1969). Translation of one of the articles on Bleomycin entitled "Treatment of lung cancer with Bleomycin". Institute of Tuberculosis and Leprosy, Tohoke University.

Oliner, H., Schwartz, R., Rubio, F., and Dameshek, W. (1961). Interstitial pulmonary fibrosis following busulphan therapy. Amer. J. Med., 31, 134.

Reid, L. (1967). The Pathology of Emphysema. Lloyd-Luke, London.

Spencer, H. (1968). Chronic interstitial pneumonia. In The Lung (Int. Acad. Path. Monographs No. 8); ed. A. A. Liebow and D. E. Smith. p. 134. Williams and Wilkins, Baltimore.

Suzuki, M., Murai, A., Watanabe, T., and Nunokawa, 0 . (1970). Treatment of cancer of the female genital organs with a new anticancer agent, Bleomycin (BLM). Eighteenth Annual Meeting of the American College of Obstetricians and Gynecologists. New York, U.S.A. 
Thompson, G. R., Schaeppi, U. H., Fleischman, R. W., Rosenkrantz, H., and Menninger, F. F., Jr. (1970). Bleomycin (NSC-125 066), toxicity of i.v. injection in dogs. Report No. MRI-USGT-70-06, Mason Research Institute, Worcester, Mass.

Tsubaki, S., Suzuki, H., and Ogawa, T. (1968), 1-10. Clinical results of Bleomycin in treatment of malignant tumors of the head and neck. Translation of paper presented at the Japanese Society of Otorhinolaryngology, 69th General Assembly.
Umezawa, H. (1965). Bleomycin and other antitumor antibiotics of high molecular weight. Antimicrob. Agents Chemother., 5, 1079.

- Ishizuka, M., Maeda, K., and Takeuchi, T. (1967). Studies on bleomycin. Cancer (Philad.), 20,891.

Maeda, K., Takeuchi, T., and Okami, Y. (1966). New antibiotics, Bleomycin A and B. J. Antibiot. (Tokyo), Ser. A, 19, 200. 